\title{
THE EVOLUTION IN THE ADMISSIONS OF YOUNG ADULTS IN A DAY HOSPITAL: COMPARISON OF TWO BIENNIUMS
}

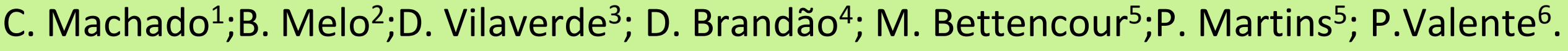
1.Psychiatry Resident;Hospital Magalhães Lemos; Portugal 3. Psychiatry Resident; Hospital de Braga, Portugal

Roughly half of all lifetime mental disorders in most studies start by the mid-teens and three-fourths by the mid-20s. Acute day hospitals are an attractive option in situations where demand for inpatient care is high and facilities exist that are suitable for conversion.

The aim of this study is to compare the proportion, as well as clinical and sociodemographic variables, of young adults (18-24 years old) admitted for treatment at day hospital in the only tertiary psychiatric hospital in the north of Portugal. The two time periods selected for comparison were 2007-2008 versus 2017-2018.

As a method, sociodemographic data and clinical data (admission motive, suicide attempts, substance abuse, diagnosis, treatment) were collected.

\section{Q RESULTS}

\section{SOCIODEMOGRAPHIC DATA:}

In 2007-2008 seven young adults

were admitted into our Day

Hospital. This number increases to

thirteen in 2017-2018.

In each biennium two young adult patients withdrew from day hospital treatment.

\section{CLINICAL DATA:}

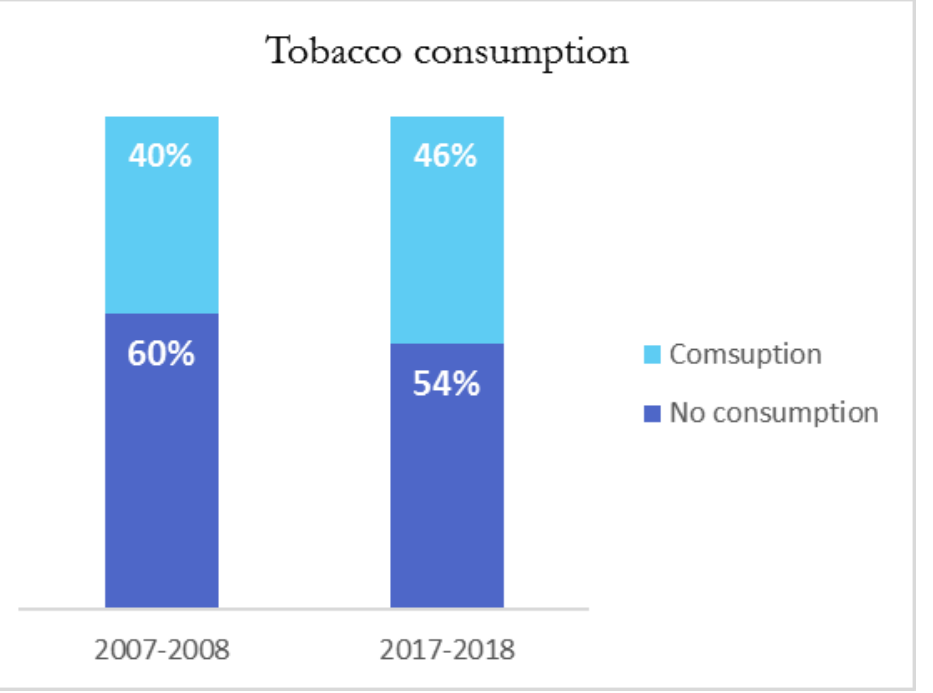

Grapich 4: The percentage of smokers increased between the two periods
Proportion of young adults/ bienniums

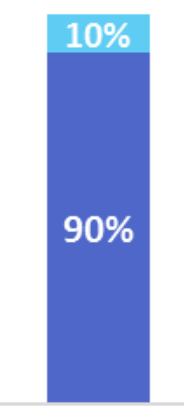

2007-2008

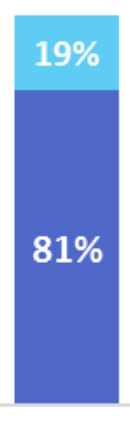

2017-2018

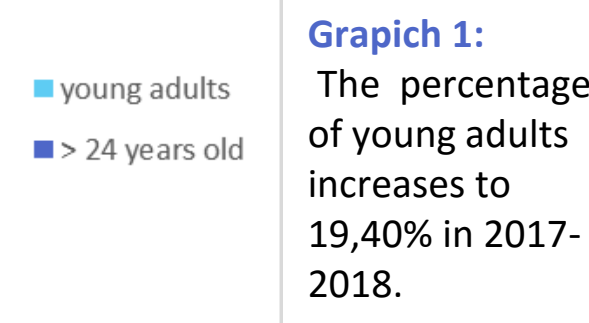

2018.
Distribution of genders in the two bienniums

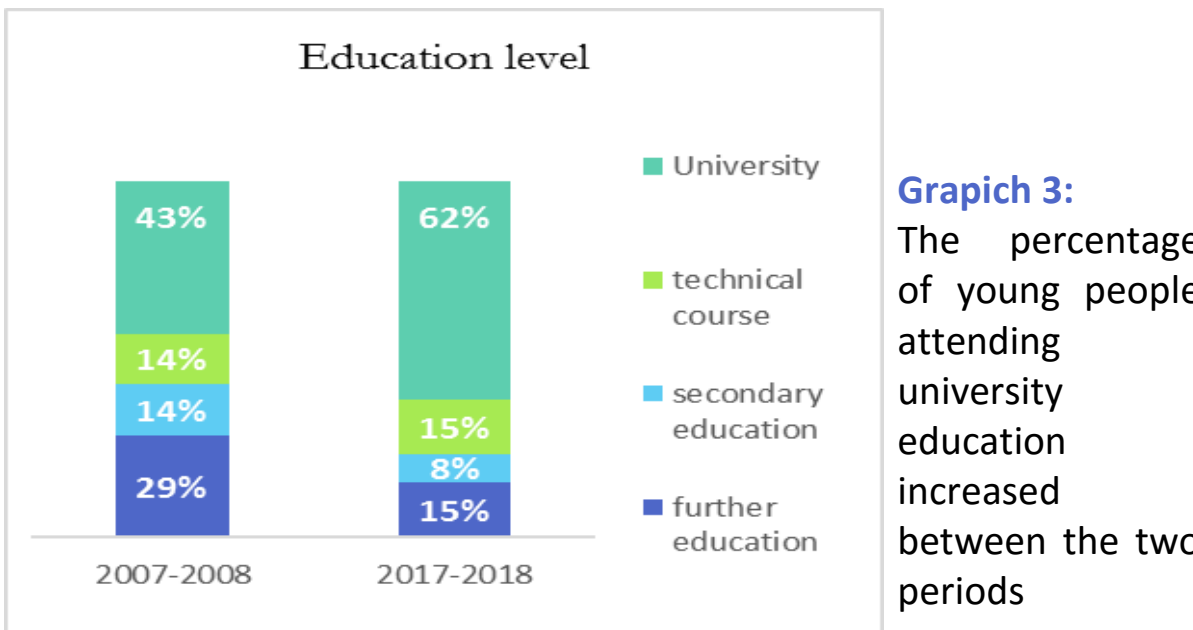

Number of drugs users by drug subtype

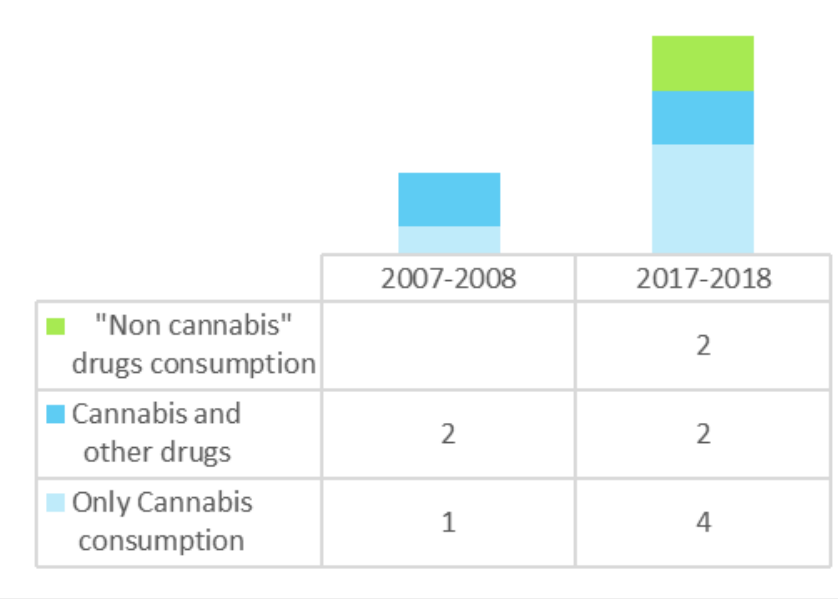

Grapich 5: The percentage of cannabis users increased between the two periods, either alone or in combination with other drugs. The most common non-cannabis drug used was alcohol

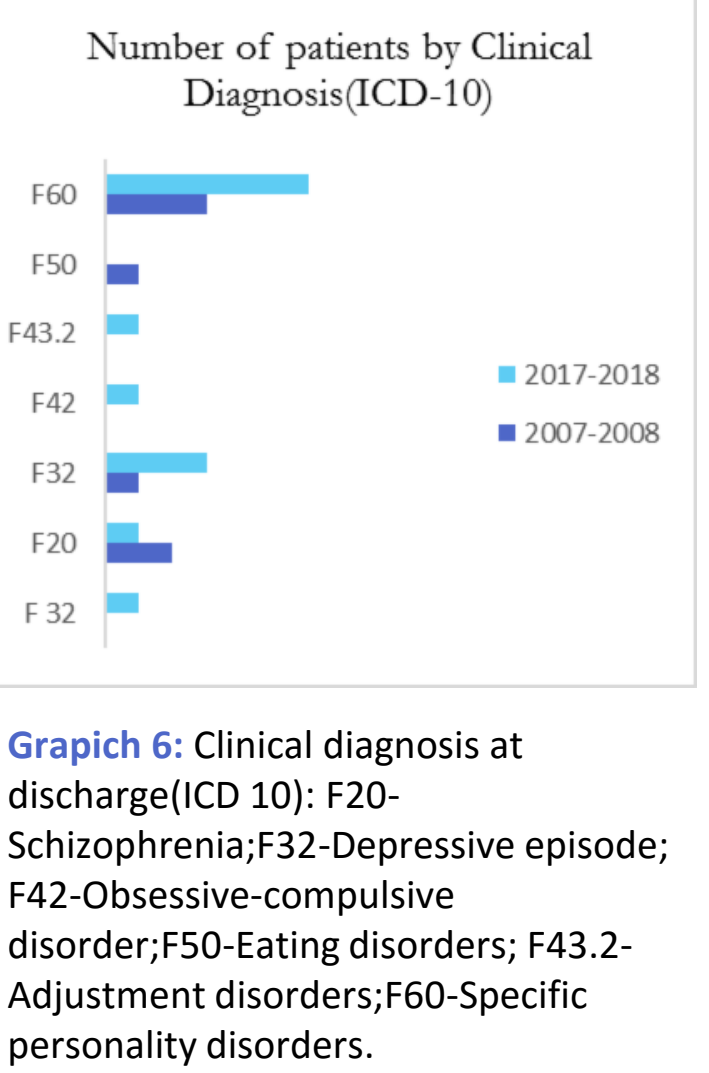

Previous admissions in inward department 2007-2008

2017-2018

Voluntary ingestion of medication

2007-2008

2017-2018

Self mutilation behaviours

2007-2008

2017-2018

Table 2: The percentage of complete admissions, voluntary ingestion of drugs and self mutilations behaviours in the two periods.

As expected the comparison of bienniums demonstrates an increase in the proportion of young adults and university students admitted to Day Hospital.Our results also show an increase in the proportion of young people with cannabis consumption and auto-injuries.

Regarding university students, the studies shown that in addition to cope with academic pressure, some students have to deal with the stressful tasks of separation and individuation from their family of origin. In this context, many experience the first onset of mental health and substance use problems or an exacerbation of their symptoms. With the increasing recognition of child and teenager mental health issues and the use of more psychotropic medications in this population, the number of young adults with mental health problems entering university is certainly increasing.

With this study we intend to highlight the increase in cases of young adults needing treatment in a more intensive regimen, such as a day hospital. Considering the early loss of social functionality in this population, the presence of school primary prevention programs in mental health becomes essential. 